\title{
Particle level set implementation on the finite volume method
}

\author{
M. Elmi \& M. Kolahdouzan \\ Department of Civil and Environmental Engineering, \\ Amirkabir University of Technology (Tehran Polytechnic), Iran
}

\begin{abstract}
Prediction of free surface elevation is one of the most important phenomena in the field of free surface flow modelling. To date different procedures have been introduced to capture the free surface. In the current study, a EulerianLagrangian Method named Particle Level Set (PLS) is developed to predict the location of free surface. The first implementation of this scheme was on the Finite Difference Method. This paper deals with the implementation of Particle Level Set on the Finite Volume mesh. IN the PLS scheme, computational particles were deployed in conjunction with LS function to raise the accuracy of function values. Results obtained from the developed model were compared with experimental and numerical results of dam break cited in the literature which represent the capability of method to predict high gradient free surface changes accurately.
\end{abstract}

Keywords: free surface flow, particle level set method, finite volume method.

\section{Introduction}

Multi-phase flow modelling is one of the most practical issues and many researchers try to implement it on their own area of expertise. Prediction of water surface elevation especially in the high gradient situations (i.e. Dam Break and Wave Breaking), is an example of multiphase flow. Different approaches have been introduced to have an accurate prediction of free surface which can be classified into two categories namely free surface tracking and free surface capturing [1].

According to free surface tracking methods, the location of free surface can be predicted by the use of Lagrangian characteristics of the flow. Although the 
implementation of these types of methods in the numerical model is simple and accurate enough in terms of modelling purposes, some limitations have been recognized for complex flows (wave reflection). Different research has been carried out using free surface tracking methods [2-5].

Interface capturing methods are based on the variation of a scalar quantity according to the advection equation. Volume of Fluid (VOF) can be classified in this category. A lot of research has been carried out according to the VOF method [6-10], each of which tries to explore the possibility and raise the accuracy of this method for free surface prediction. Osher and Fedkiw [11] introduced another method in this category called Level Set (LS). This method is not satisfying mass conservation even with the high resolution techniques [12], Therefore it is not so popular in Computational Fluid Dynamics. Different researchers try to combine methods to overcome the problem which was existing in each one individually. Sussmam and Puckett [13] tried to combine LS and VOF methods. In recent years an attempt has been made to improve the LS method in a way that it can be used for free surface prediction. Particle Level Set (PLS) is the result of recent researches in this field [14, 15]. The first implementation of the PLS Scheme was on the Finite Difference method. In this research an attempt has been made to implement the PLS method in the Finite Volume Method mesh.

\section{Governing equations}

Governing equations of incompressible viscous fluid flow are expressed by the Navier-Stokes equations which can be shown as follows [1]:

$$
\begin{gathered}
\frac{\partial \boldsymbol{u}}{\partial t}+\boldsymbol{u} \cdot \boldsymbol{\nabla} \boldsymbol{u}=v \nabla^{2} \boldsymbol{u}-\frac{1}{\rho} \nabla P+S \\
\nabla \cdot \mathrm{u}=0
\end{gathered}
$$

where: $\boldsymbol{u}$ is the velocity vector, $v$ is the fluid viscosity, $P$ is the pressure and $S$ is the body forces.

To discretize governing differential equations based on the Finite Volume method, it is essential to integrate these equations over the control volume. In the current study, discretization of advection and viscous terms in equations (1) were carried out by the use of a central difference scheme (CDS). In order to prevent non-physical oscillation in the pressure field due to CDS application, it was suggested to use a special interpolation scheme for advection acceleration terms in the momentum equation which can be shown as [16]:

$$
\boldsymbol{u}_{f}=L I(\boldsymbol{u})+\frac{\Delta t}{\rho} \frac{\partial P}{\partial \boldsymbol{n}}
$$

where: $L I(\boldsymbol{u})$ is the linear interpolation of velocity components, $\Delta t$ is the time step and $\boldsymbol{n}$ is the normal vector to control volume face.

In order to couple momentum and continuity equations, a Fractional Step method was applied [17]. In the first step, a momentum equation was solved to 
obtain velocity components in the absence of pressure terms (mid-term velocities):

$$
\frac{\widetilde{u}_{i}-u_{i}^{n}}{\Delta t}+\frac{\partial u_{i} u_{j}}{\partial x_{j}}=v \frac{\partial^{2} u_{i}}{\partial x_{j} \partial x_{j}}+S_{i}
$$

In order to include pressure effect on the velocity components in the new time step the following relationship was used [17]:

$$
u_{i}^{n+1}=\tilde{u}_{i}-\frac{1}{\rho} \frac{\partial p^{n+1}}{\partial x_{i}}
$$

By which can be reformulated as equation (5):

$$
\nabla^{2} p^{n+1}=\rho \boldsymbol{\nabla} \cdot \widetilde{\boldsymbol{u}}
$$

Equation (5) is a Poisson type equation which was used to obtain pressure field in the study area. Solution algorithm for governing equations of fluid flow can be summarized as:

1) Solving equation (3) to obtain mid-term velocity components $(\widetilde{\boldsymbol{u}})$.

2) Equation (5) was solved to obtain pressure field.

3) To correct the velocity components values equation (4) was deployed.

To satisfy conservation of momentum, it is essential to correct velocity components obtained from step (1) in step (3) using pressure gradient. In multiphase flow, the discretization procedure for Poisson equation is very important as the density of fluid is suddenly changes on the free surface. In the current study to discretize Poisson equation, the broken line method was deployed [18]. According to broken line method (Figure 1), in free surface grids, fluid density was calculated by the following relationship [18]:

$$
\rho=\alpha \rho_{1}+(1-\alpha) \rho_{2}
$$

where: $\alpha$ is the volumetric ratio of two fluids and $\rho$ is the fluid density. The pressure values in the side centre of each computational grid can be evaluated by equation (7):

$$
P_{f}=\frac{P_{A} \rho_{B} \delta_{B}+P_{B} \rho_{A} \delta_{A}}{\rho_{B} \delta_{B}+\rho_{A} \delta_{A}}
$$

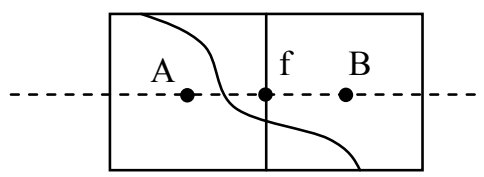

Figure 1: $\quad$ Broken line discretization method in each computational grid cell. 


\section{Particle level set method}

The level set function magnitude in each computational point is the smallest distance between free surface and that point. In addition, this function has different sign in each phase of fluid (Figure 2). Computational particles were deployed in conjunction with LS function to raise the accuracy of function values [11].

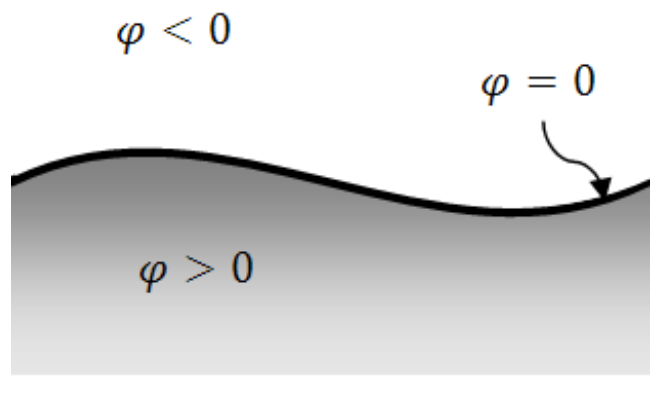

Figure 2: $\quad$ LS function.

The level set function has different geometrical properties like as [11]:

$$
\begin{gathered}
|\nabla \varphi|=1 \\
\boldsymbol{n}=\frac{\boldsymbol{N}}{|\boldsymbol{N}|}=\frac{\boldsymbol{\nabla} \varphi}{|\boldsymbol{\nabla} \varphi|}=\boldsymbol{\nabla} \varphi
\end{gathered}
$$

where: $\boldsymbol{n}$ is the normal vector to the free surface and $\varphi$ is the LS function. The governing equation of LS function is the advection type and can be written as [11]:

$$
\frac{\partial \varphi}{\partial t}+\boldsymbol{u} \cdot \boldsymbol{\nabla} \varphi=0
$$

In order to transfer the LS function in the PLS method, a semi-Lagrangian approach was used. According to this approach, to obtain the new value for the LS function in each grid cell, it is essential to assume that the cell centre was moved equal to $-\boldsymbol{u} . \Delta t$ and the LS value in this new position was considered as the LS value for the specified grid cell. In the current study, LS function gradient have been calculated through equation (11):

$$
\boldsymbol{\nabla} \varphi=\frac{\sum_{\text {faces }} \varphi_{f} \boldsymbol{n}_{f} A_{f}}{V}
$$

where: $A_{f}$ is the computational cell area, $V$ is the cell volume and $\boldsymbol{n}_{f}$ is the unit vector normal to the surface. 
It is essential to point out that the LS function is solved in a very thin band close to the free surface. Computational errors where produced in the transport of each LS function, which in turn cause uncertainty on the free surface position. Many techniques were introduced to overcome this problem [11]. In the current study, the Fast Marching method was deployed to reduce inaccuracy in the prediction of free surface elevation [19].

Computational particles were used to correct the LS function transfer in the computational domain. These particles are distributed close to the initial free surface in a way that each particle radius introduces the distance of particle and free surface. In addition, the radius of each particle can be positive or negative according to its placement in different phase. To obtain more accurate results, these particles can have overlap in terms of their placement near the water surface (Figure 3). In Figure 3 the hatched area represents the difference between the real situation and model prediction for a straight line.

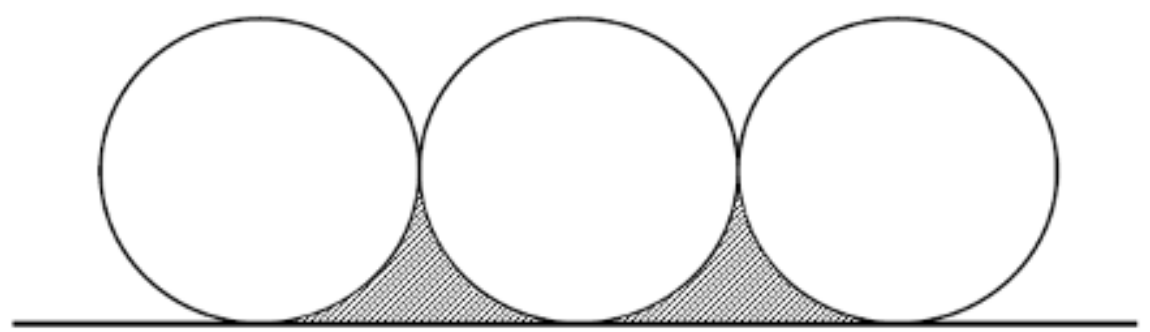

(a) Three particles without overlap

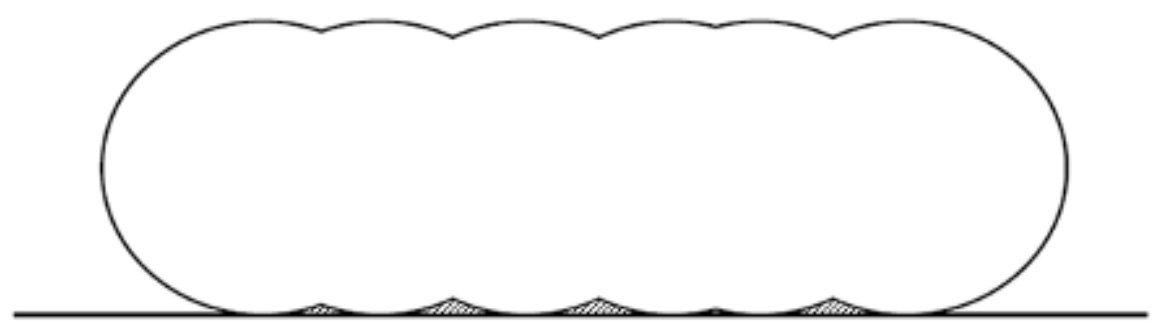

(b) Six particles with overlap

Figure 3: $\quad$ Illustration of a straight line with particles.

In each time step, the placement of each particle was calculated by the $3^{\text {rd }}$ order TVD-Runge Kutta method and then, the value of the LS function was corrected with these particles. The step by step method of correction can be summarized as [14]: 
1) Those particles which have distance more than their radius outside of their own fluid phase with free surface, considered as an escaped particle. These particles are used to correct the free surface position.

2) By generating a mesh for computational domain, the placement of each particle in the grid generation was investigated and the value of LS function for surrounding grid cells was calculated as follows:

$$
\varphi_{p}=r_{p}-\left|\boldsymbol{x}-\boldsymbol{x}_{p}\right| \cdot \operatorname{sgn}\left(r_{p}\right)
$$

3) LS function is corrected according to equations (13)-(15):

$$
\begin{gathered}
\varphi^{+}=\max \left(\varphi_{p}, \varphi_{\text {old }}\right), \forall p \in E^{+} \\
\varphi^{-}=\min \left(\varphi_{p}, \varphi_{\text {old }}\right), \forall p \in E^{-} \\
\varphi_{\text {new }}=\left\{\begin{array}{l}
\varphi^{+} \text {if }\left|\varphi^{+}\right| \leq\left|\varphi^{-}\right| \\
\varphi^{-} \text {if }\left|\varphi^{+}\right|>\left|\varphi^{-}\right|
\end{array}\right.
\end{gathered}
$$

where $E^{+}$is all positive-radius escaped particles and $E^{-}$is all negative-radius escaped particles. It is essential to point out that the difference between above method and main PLS method is on the selection of surrounding mesh cells in order to correct LS function. It is worth to point out that this method can be used on the unstructured finite volume mesh which raised the capability of the method for using in complex geometry.

\section{Model verification}

In order to represent capability of model to represent free surface elevation, a dam break problem has been chosen (Figure 4). The uniform 100*75 grid mesh with $0.0001 \mathrm{sec}$ time step has been used for modelling purposes. A no-slip boundary condition was applied for all of solid boundaries. Initial free-surface elevation and initial particle distribution are shown in figure 5. The predicted model results for free surface elevation and also experimental measurements are presented in figure 6. From the comparison of predicted and measured values for free surface elevation, it can be concluded that the developed model which consists of the PLS approach can well predict the free surface. In addition model results were compared with experimental results and other numerical models cited in the literature (Figure 7). From figure 7 it can be concluded that in the early stages of modelling, the numerical model results based on the Lagrangian approach are more accurate while close to the end of modelling simulation time, the LS approach is more accurate in terms of predicting free surface elevation. 


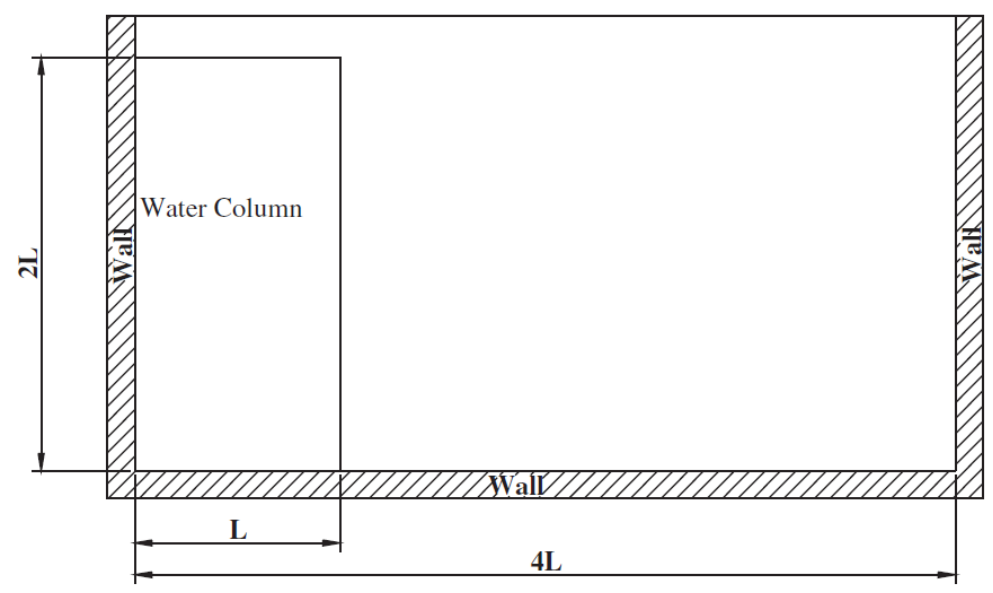

Figure 4: Geometry of dam break problem.

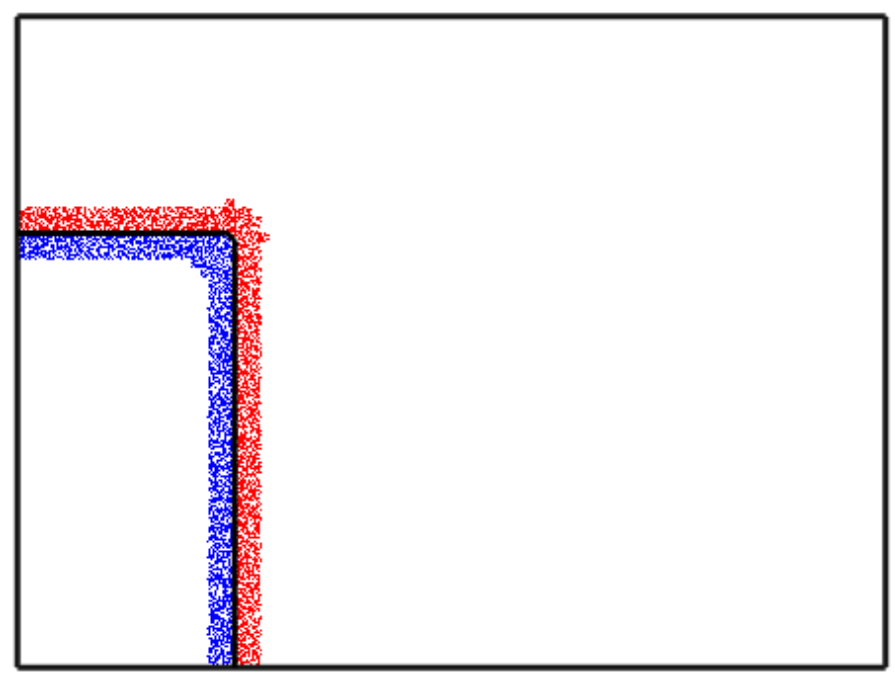

Figure 5: Initial particle distribution in dam break problem. 
Present Study

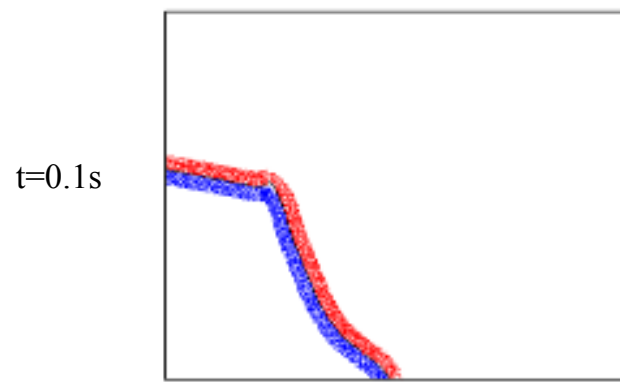

Empirical Results

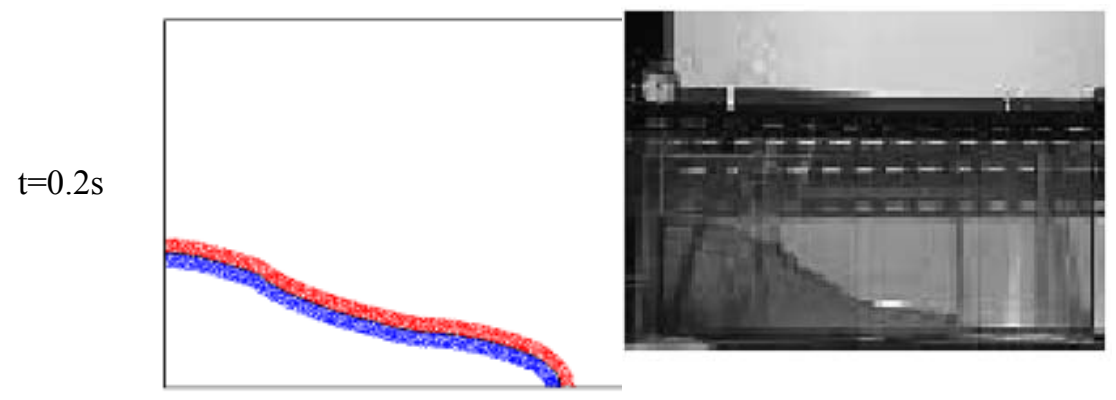

$\mathrm{t}=0.3 \mathrm{~s}$

$\mathrm{t}=0.4 \mathrm{~s}$
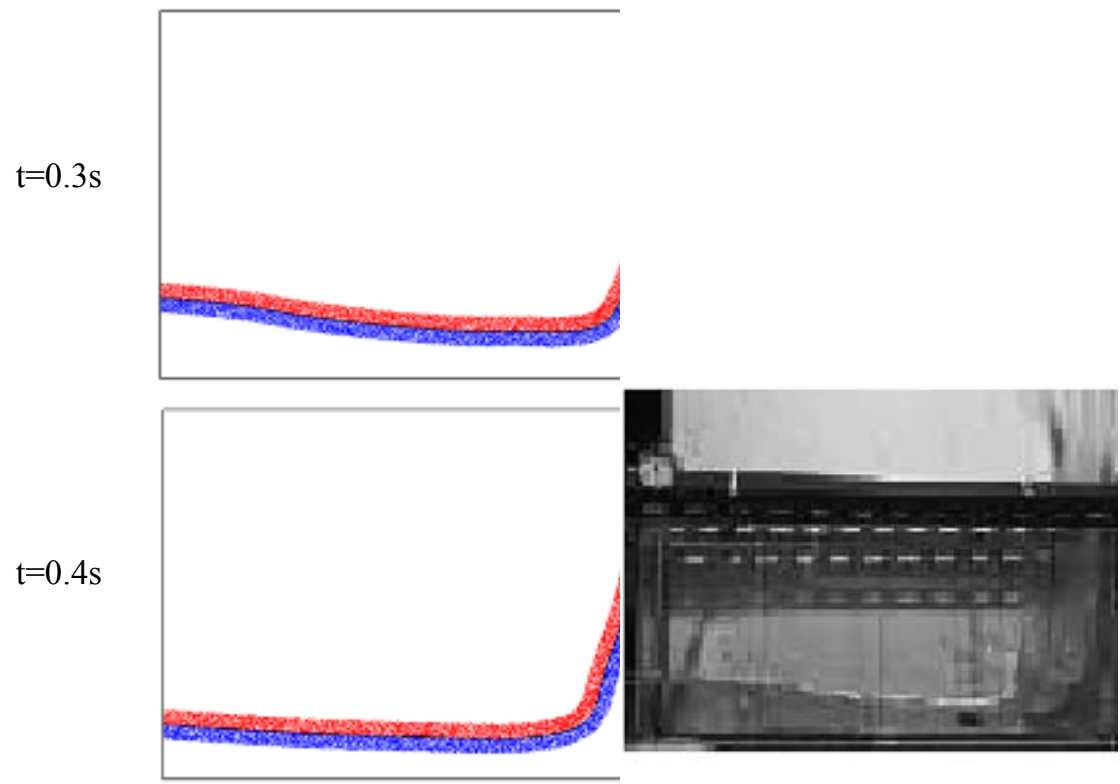

Figure 6: $\quad$ Free surface variation and computational particles. 


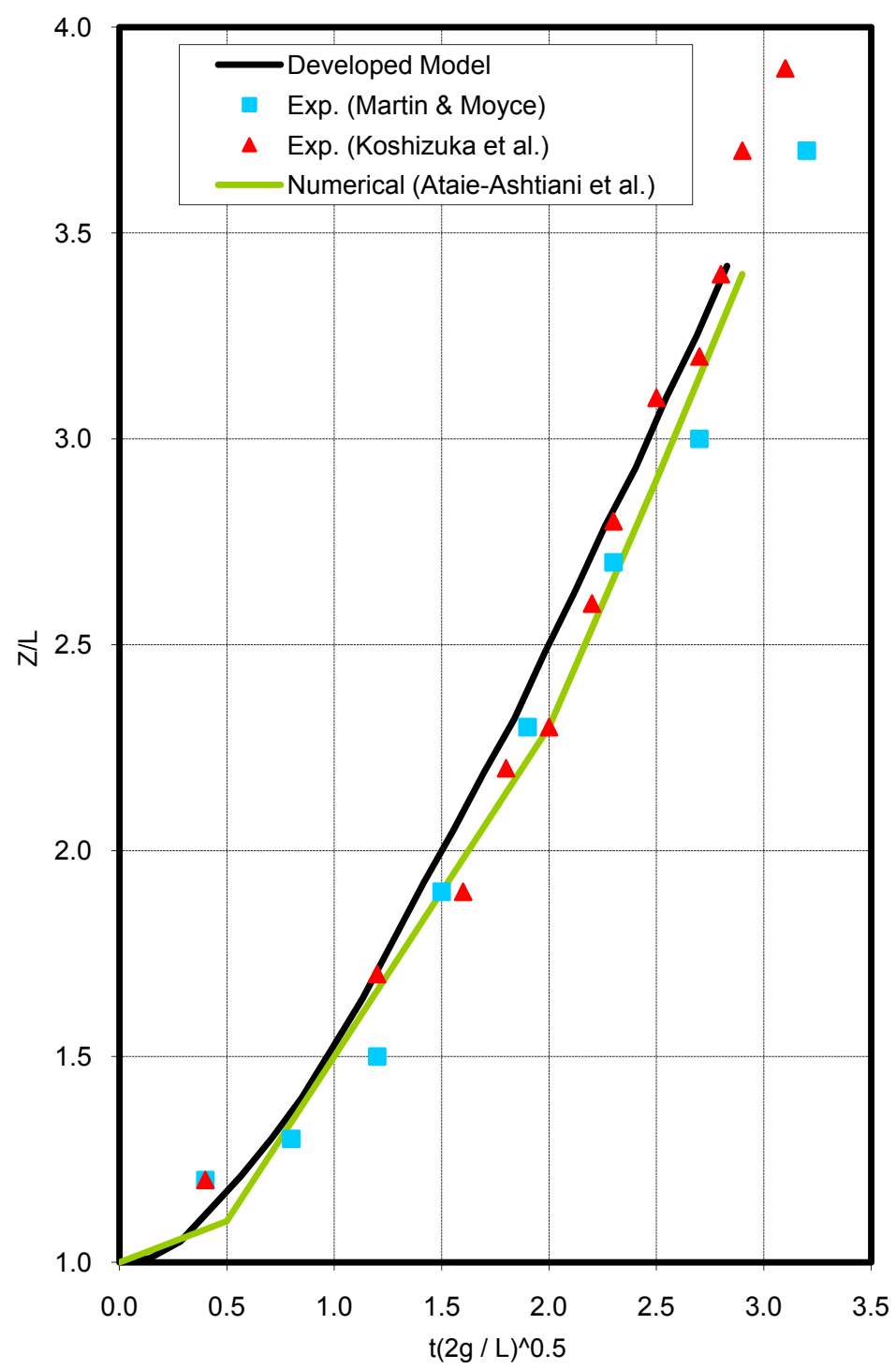

Figure 7: Comparison of measured and predicted values of water in front.

\section{Conclusion}

Free surface prediction is one of the most important issues in the field of fluid dynamics. The problem is more critical in coastal area were the short waves are the most dominant hydrodynamic phenomena. In this research study the PLS 
method has been chosen to investigate its capability on the free surface prediction. Results obtained from the developed model show that the PLS method can well predict the free surface elevation in comparison with measurements and other numerical models cited in the literature.

\section{References}

[1] Ferziger, J., Peric, M., 1996. Computational methods for fluid dynamics. Springer Verlag.

[2] Raad, P., Chen, S. and Johnson, D., 1995. The Introduction of Micro Cells to Treat Pressure in Free Surface Fluid Flow Problems. J. Fluids Eng. 117, 683-690.

[3] Torres, D. and Brackbill, J., 2000. The Point-Set Method: Front Tracking without Connectivity. J. Comp. Phys. 165, 620-644.

[4] Tryggvason, G., Bunner, B., Esmaeeli, A., Juric, D., Al-Rawahi, N., Tauber, W., Han, J., Nas, S. and Jan, Y.-J., 2001. A Front-Tracking Method for the Computations of Multiphase Flow. J. Comp. Phys. 169, 708-759.

[5] Unverdi, S.-O. and Tryggvason, G., 1992. A Front-Tracking Method for Viscous, Incompressible, Multi-fluid Flows, J. Comp. Phys. 100, 25-37.

[6] Ubbink, O., Issa, R. I., 1999. A method for capturing sharp fluid interfaces on arbitrary meshes. J. of computational physics 153, 26-50.

[7] Puckett, E., Almgren, A., Bell, J., Marcus, D. and Rider, W., 1997. A HighOrder Projection Method for Tracking Fluid Interfaces in Variable Density Incompressible Flows. J. Comp. Phys. 130, 269-282.

[8] Hirt, C. and Nichols, B., 1981. Volume of Fluid (VOF) Method for the Dynamics of Free Boundaries. J. Comp. Phys. 39, 201-225.

[9] Williams, M., Kothe, D. and Puckett, E., 1999. Approximating Interfacial Topologies with Applications for Interface Tracking Algorithms. 37th AAIA Aerospace Sciences Meeting, 99-1076, AIAA.

[10] Williams, M., Kothe, D. and Puckett, E., 1999. Convergence and Accuracy of Kernal-Based Continuum Surface Tension Models. Fluid Dynamics at Interfaces, pp. 347-356, Cambridge University Press.

[11] Osher, S., Fedkiw, R., 2002. Level Set Methods and Dynamic Implicit Surfaces, Springer.

[12] Jiang, G.-S. and Peng, D., 2000. Weighted ENO Schemes for HamiltonJacobi Equations. SIAM J. Sci. Comput 21, 2126-2143.

[13] Sussmam, M. and Puckett, E., A Coupled Level Set and Volume of Fluid Method for Computing 3D and Axisymmetric Incompressible Two-Phase Flows, J. Comp. Phys. 162, 301-337 (2000).

[14] Enright, D., Fedkiw, R., Ferziger, J. and Mitchell, I., 2002. A Hybrid Particle Level Set Method for Improved Interface Capturing. J. Comput. Phys. 183, 83-116.

[15] Enright, D., Losasso, F. and Fedkiw, R., 2005. A Fast and Accurate SemiLagrangian Particle Level Set Method. Computers and Structures 83, 479490. 
[16] Zang, Y., Street, R.L. and Koseff, J.R., 1994. A non-staggered fractional step method for time-dependent incompressible Navier-Stokes equations in curvilinear Coordinate. J. of computational physics 114, 18-33.

[17] Kim, D., Choi, H., 2000. A second-order time-accurate finite volume method for unsteady incompressible flow with hybrid unstructured grids. J. of computational physics 162, 411-428.

[18] Jahanbakhsh, E., Panahi, R., Seif, M.S., 2007. Numerical simulation of three-dimensional interfacial flows. International Journal of Numerical Methods for Heat \& Fluid Flow 17, 384-404.

[19] Tsitsiklis, John N., 1995. Efficient Algorithms for Globally Optimal Trajectories. IEEE TRANSACTIONS ON AUTOMATIC CONTROL 40.

[20] Ataie-Ashtiani, B., Farhadi, L., 2006. A stable moving-particle semiimplicit method for free surface flows. Fluid Dynamics Research 38, 241256

[21] Koshizuka, S., Nobe, A., Oka, Y., 1998. Numerical analysis of breaking waves using the moving particle semi-implicit method. Int. J. Numer. Methods Fluids 26, 751-769.

[22] Martin, J.C., Moyce, W.J., 1952. An experimental study of the collapse of liquid columns on a rigid horizontal plane. Philos. Trans. R. Soc. London Ser. A, 244-312. 